\title{
7 Summaries in English, German and Russian
}

This scientific-archaeological study focused on the reconstruction of mobility and diet in the West Eurasian steppes in the period between 3500 and $300 \mathrm{BC}$ using ${ }^{87} \mathrm{Sr} /{ }^{86} \mathrm{Sr}, \delta^{18} \mathrm{O}, \delta^{15} \mathrm{~N}$ and $\delta^{13} \mathrm{C}$ analyses. It was a subproject of research group A II Spatial effects of technological innovations and changing ways of life within the framework of the Excellence Cluster Topoi. The study covered a vast area that has been subdivided into representative micro-regions or single sites, namely Bulgaria and Hungary in the Carpathian-Balkan region, central and eastern Ukraine in the North Pontic region, the sites in the Kuban region, the northwest Caspian steppe and in the Middle Volga region in Russia and one site in Kazakhstan, in Central Asia. Samples were selected from sites dating to the Eneolithic, the Early and Middle Bronze Age and the Iron Age and can be associated with four time periods; the late Eneolithic in the second half of the $4^{\text {th }}$ millennium BC, the Early Bronze Age Yamnaya culture that dominates the first half of the 3 rd millennium BC and the subsequent Catacomb culture, which can be dated to the second half of the 3 rd millennium BC as well as cultural groups of the Scythian period in the Ist millennium BC. Tooth and bone samples were selected for multi-isotope analysis, i.e. ${ }^{87} \mathrm{Sr} /{ }^{86} \mathrm{Sr}, \delta^{18} \mathrm{O}, \delta^{15} \mathrm{~N}$ and $\delta^{13} \mathrm{C}$ analyses. The basic questions concerned the mobility and dietary patterns of these cultural groups. It was also interesting to see whether differences in stable isotope ratios could be identified in the periods immediately before and after $3000 \mathrm{BC}$, which is when specialised cattle husbandry emerged and means of transport like wagons or wagon parts appeared as grave furniture. Furthermore, the widespread and highly consistent burial traditions in the North Pontic and adjacent regions imply wide-ranging cultural contacts. Changes in climate are thought to have occurred at this time, with palaeoclimatic studies suggesting that a period of increasing aridity started around the late 4 th millennium and is evident throughout the whole 3 rd millennium BC. All this might have led to shifts in lifestyle with an increased degree of mobility. As a basis for comparison, samples were taken dating to the Scythian period in the Ist millennium BC, a time associated with the emergence of fully-fledged nomadism and mounted warfare and therefore implying highly developed mobility.

The introduction of the study aims as well as the geographical and chronological attribution of the study sites in chapter I are followed by the description of the archaeological cultures related to the sampled human individuals, an overview of the applied methods and an introduction to the choice of sampling material in chapter 2 . In chapter 3 , the sample sites are briefly described. The selection of sites, in which the author was not involved, followed the principle of gaining a representative cross-section of the study region. It was unfortunately not taken into consideration that sample sizes per site and microregion should be statistically relevant and a numerical consistency of sex and age and chronological groups was not attempted. These factors complicated the evaluation of the data significantly.

The study was separated into two main sections; the first focused on mobility and migration of the steppe people in the periods mentioned above, while the second dealt with their dietary behaviour. The mobility and migration study included the analysis of ${ }^{87 \mathrm{Sr}} /{ }^{86} \mathrm{Sr}$ and $\delta^{18} \mathrm{O}$ in tooth enamel samples from I42 human individuals. The use of $\delta^{15} \mathrm{~N}$ and $\delta^{13} \mathrm{C}$ analysis on the bone of 54 human individuals from selected sites in the West Pontic, the North Pontic and the Kuban region provided the basis of the palaeodietary study. These two studies are regarded as separate investigations due to varying sample bases.

Mobility theories are frequently mentioned but not often included in archaeological research. In this study common theories of mobility and migration were introduced and discussed at the beginning 
of chapter 4. Their application to the study region was relatively complicated, since the cultures being examined here are associated with a number of elements of uncertainty. The definition of the boundaries between the Eneolithic steppe cultures and the regional variants of the Early Bronze Age is rather vague and disputed in archaeological research. Their economies are heavily disputed and can only be regarded as being primarily based on specialised cattle husbandry that implied some kind of increased mobility. The mobility ranges and their directions, however, remain highly speculative. Settlement sites are rare and even less frequently archaeologically investigated. Temporary, probably seasonal, camps are sometimes indicated, for example in the northwest Caspian region, but there is also evidence of settlements that were permanent or at least occupied for long periods, for example along the lower Dnieper. References for possible migration cycles, for example circular or resource-related ones, do not exist. Furthermore, the last places of residence of supposedly mobile communities do not necessarily equate to the burial places. This issue questions the value of collecting reference samples to establish the 'local' isotopic signatures in the burial mound environment.

Both ${ }^{87} \mathrm{Sr} /{ }^{86} \mathrm{Sr}$ and $\delta^{18} \mathrm{O}$ analyses depend on varying factors; this is basically geology for ${ }^{87} \mathrm{Sr} /{ }^{86} \mathrm{Sr}$ and multiple factors like amount of precipitation, temperature, distance from the ocean, altitude and latitude for $\delta^{18} \mathrm{O}$. The combination of ${ }^{87} \mathrm{Sr} /{ }^{86} \mathrm{Sr}$ and $\delta^{18} \mathrm{O}$ analyses led to high-quality results, which enabled the identification of micro-regions, including some overlaps, and simplified the identification of outlier values. The results of the strontium isotope analysis varied; Consistent ${ }^{87} \mathrm{Sr} /{ }^{86} \mathrm{Sr}$ values were achieved for sites like Benkovski (Bulgaria), Nevskoe (Ukraine) and Olennii (Russia), whereas other sites such as Ovchartsi (Bulgaria), Kirovograd (Ukraine) and the sites in the Middle Volga region (Russia) showed much more variability in the ${ }^{87} \mathrm{Sr} /{ }^{86} \mathrm{Sr}$ values. These patterns were not reflected in the $\delta^{18} \mathrm{O}$ values, however. Sites featuring homogeneous ${ }^{87} \mathrm{Sr} /{ }^{86} \mathrm{Sr}$ values showed more variable $\delta^{18} \mathrm{O}$ values, for example Vinogradnoe and Nevskoe (both Ukraine), while sites with divergent ${ }^{87} \mathrm{Sr} /{ }^{86} \mathrm{Sr}$ values had less varied $\delta^{18} \mathrm{O}$ values, e.g. Kirovograd (Ukraine). The combination of both isotopic systems made it possible to distinguish between different mobility patterns. Nevertheless, the question of whether these differences are related to larger mobility ranges, to different social organisation or to varying data sets remains unexplained. Similar isotopic results were repeatedly associated with the use of the kurgans over just one cultural period, perhaps equivalent to one single community, for example in Nevskoe (Ukraine), Olennii and Sukhaya Termista (Russia), while widely distributed isotopic results often correlated to a diachronic use of the burial mounds, for example in Kirovograd and Peshtchanka (Ukraine). Nevertheless, there were also contradictory examples. Intra- and interregional comparisons were complicated by varying strategies in reference sampling. Regardless of the varying levels of reliability of the reference samples, it was suggested that the data for the Eneolithic and the Yamnaya period in the western study areas were more widely dispersed than the isotope values of other regions. Only small ranges were evident for the Early Catacomb culture. Strontium and oxygen isotope patterns seemed to marginally shift over time, which might be associated with the beginning of a period of increasing aridity and/or an economic change and the appearance of partly mobile pastoralism at the end of the $4^{\text {th }}$ millennium BC. No higher degree of mobility could be identified isotopically for unusual graves like wagon burials.

The relatively restricted ranges of isotope values from the Scythian samples from the North Pontic sites can be interpreted as limited or non-detectable mobility. Alternatively, it could be that movement is not visible isotopically because of the homogeneous nature of expected isotope ratios across this wideranging region. The different locations of the sites and the isotopic variations seen within some sites argue against interpreting the sampled Scythians of the North Pontic as one residence community. A higher degree of mobility, perhaps suggesting patterns of transhumance, were indicated by larger 
${ }^{87} \mathrm{Sr} /{ }^{86} \mathrm{Sr}$ and $\delta^{18} \mathrm{O}$ ranges and standard deviations in the humans and horses from the Kazakh Altai Mountains, also dating to the Scythian period. A case study on a triple cattle deposition from Zauschwitz (Germany) affiliated to the Globular Amphora culture, which has chronological and economic parallels to the Yamnaya culture, showed differing animal movement patterns, some of which also suggested transhumance. The application of ${ }^{87} \mathrm{Sr} /{ }^{86} \mathrm{Sr}$ laser ablation in this context gave excellent results and strengthened the interpretation of regular mobility.

The data obtained from ${ }^{87} \mathrm{Sr} /{ }^{86} \mathrm{Sr}$ and $\delta^{18} \mathrm{O}$ analyses neither absolutely confirmed nor contradicted possible migrations into the regions west and southwest of the distribution areas of larger groups of the Eneolithic steppe cultures and the Early Bronze Age cultures of the North Pontic region. The ${ }^{87} \mathrm{Sr} /{ }^{86} \mathrm{Sr}$ and $\delta^{18} \mathrm{O}$ results of several individuals from Hungarian burial mounds indicated the presence of migrants, which complied with the archaeology. The dispersed ${ }^{87} \mathrm{Sr} /{ }^{86} \mathrm{Sr}$ and $\delta^{18} \mathrm{O}$ values of some individuals from the Bulgarian sites, which would agree with values expected for the North Pontic region, also corresponded with the archaeological evidence and inferred immigration. The data obtained from ${ }^{87} \mathrm{Sr} /{ }^{86} \mathrm{Sr}$ and $\delta^{18} \mathrm{O}$ isotope analyses did not indicate foreign origins for other individuals that are also buried according to North Pontic burial traditions. Due to a relatively small data set and the lack of a good archaeological characterisation of the contemporaneous local burial practices, further analyses are needed to support or discard suggested migrations.

In addition to the investigations into mobility and migration, a palaeodietary study using $\delta^{\mathrm{I}} \mathrm{C}$ and $\delta^{15} \mathrm{~N}$ analysis was conducted on human skeletal material that derived from three sites in Bulgaria, four sites in Ukraine and one site in the Kuban region. Statistical tests proved a correlation between $\delta^{\mathrm{I} 3 C}$ and $\delta^{15} \mathrm{~N}$, which coincided with the geographical locations and their climatic conditions. Besides $\mathrm{C}_{3}$ terrestrial herbivores and plants providing the dietary basis of all sampled human individuals, the West Pontic, i.e. the Bulgarian, samples were distinctly different to the samples deriving from the North Pontic and the Kuban region. A shift of approximately $3 \%$ in $\delta^{15} \mathrm{~N}$ values between the herbivore bones from the Bulgarian and Ukrainian sites in reference studies and the present study versus the herbivore data from south Russia was observed, which can best be explained by the differing climatic conditions. The prevalence of significantly higher $\delta^{15} \mathrm{~N}$ values among humans from the North Pontic and the Kuban region compared to Bulgaria might additionally be the result of the dietary addition of freshwater fauna by the North Pontic steppe people during the Eneolithic and Bronze Age. In concurrence with earlier light stable isotope studies in the West Eurasian steppes, it was suggested that fishing and the exploitation of freshwater resources were major subsistence activities during these periods. Evidence of fishing activities for the Bulgarian humans, on the contrary, was slight to non-existent but the isotope signatures of the Early Bronze Age human individuals in this region hinted to an increased impact of $\mathrm{C}_{4}$ plants. This is potentially due to changes in agricultural activities such as the cultivation of $\mathrm{C}_{4}$ plants like millet. In the North Pontic region, on the other hand, there is no clear evidence of millet consumption or production in the Early Bronze Age and it only starts to play an important role in the later Bronze Age and subsequent Iron Age. Statistical tests were applied with severe limitations as group sample sizes were mostly too small. Chronological variations were statistically proven between the Yamnaya and the Early Catacomb cultures and between the Early and the Developed Catacomb cultures at Vinogradnoe. There was, however, no decisive chronological trend. Marginal variations existed between children and adults, which might be indicators for a meat- or fish-enriched diet in adulthood, while no variations associated with differences in sex could be detected. In conclusion, the large-scale palaeodietary investigation enabled the detection of dietary changes as well as changes based on climate variations associated with geographical and/or chronological changes between the West Pontic, the North Pontic and the 
Kuban region. The consumption of $\mathrm{C}_{3}$ terrestrial herbivores and domesticate products provided the most important dietary component but the diet of the steppe communities during the Eneolithic, the Early and Middle Bronze Age probably had more facets than only herbivore meat, namely freshwater resources and plants. In addition to a minor impact of fish consumption, the most probable explanation for the differences between the West and the North Pontic data is climatic variations and differences in forest cover. A more profound insight into the diet of the Eneolithic and Bronze Age individuals in the investigated regions would require the extension of the sample sizes of both humans and animals and the establishment of a reconstructed food web, which would further provide the basis for the development of mixing models.

It became apparent that the sampling of such a vast study area is extremely complicated and sampling strategies certainly need to follow the same standards as in the investigation of single sites. Statistically relevant sample sizes of human individuals as well as food and water sources and the isotopic characterisation of the immediate environs are required to enable clear conclusions. Therefore, as a substitute for large-scale random sampling, it is suggested that a few kurgan sites be selected, where diachronic analysis and a profound and systematic characterisation of the environment would be possible. It could be shown that the study area is isotopically distinguishable, however, which would provide a sound base for future studies using stable isotope analysis in the same study area. Although questions about mobility and dietary patterns and migrations of the Eneolithic, Early and Middle Bronze Age and Iron Age individuals in the West Eurasian steppes could only be answered speculatively rather than conclusively, this study nevertheless provides an excellent starting point for further isotope work.

Die vorliegende Studie vereinte Methoden aus der Archäologie und den Naturwissenschaften um das Mobilitäts- und Ernährungsverhalten der Menschen in der Westeurasischen Steppe im Zeitraum zwischen 3500 und 300 v. Chr. zu beleuchten. Es handelte sich dabei um ein Subprojekt der Forschergruppe A II Spatial effects of technological innovations and changing ways of life des Exzellenzclusters Topoi. Im Rahmen der Studie wurden repräsentative Mikroregionen sowie einzelne Fundorte im KarpatenBalkan-Raum (in Bulgarien und Ungarn), im Nordpontikum (im südlichen Bereich der zentralen Ukraine und in der Ostukraine), in Russland (im Kuban-, dem nordwestlichen Kaspigebiet und in der Mittleren Wolgaregion) sowie im kasachischen Altai ausgewählt. Aus den genannten Regionen wurden menschliche Individuen selektiert, die in die zweite Hälfte des 4. Jahrtausends v. Chr., in das 3. sowie das I. Jahrtausend v. Chr. datieren, und den äneolithischen Steppenkulturen, den bronzezeitlichen Jamnaja- und Katakombengrabkulturen sowie Kulturgruppen aus der skythischen Periode zugerechnet werden. An ihren Zähnen und Knochen wurden Multi-Isotopenanalysen, genauer ${ }^{87} \mathrm{Sr} /{ }^{86} \mathrm{Sr} r, \delta^{18} \mathrm{O}-$, $\delta^{15} \mathrm{~N}$ - und $\delta^{13} \mathrm{C}$-Analysen, durchgeführt. Neben der Evaluation der Ergebnisse für die einzelnen Fundorte und dem Vorschlag möglicher Mobilitäts- und Ernährungsmuster dieser Gemeinschaften, stand die Fragestellung im Fokus, ob sich im Zusammenhang mit Innovationsprozessen eine erhöhte Mobilität feststellen lässt, wofür die Zeit vor und nach ca. 3000 v. Chr. auf mögliche Unterschiede in den Verteilungen der Isotopenverhältnisse untersucht wurde. In Zusammenhang mit der Jamnajakultur fand ungefähr an der Wende vom 4. zum 3. vorchristlichen Jahrtausend ein Übergang zu einer auf Rinder spezialisierten Viehhaltung statt und Fortbewegungsmittel wie Wagen bzw. deren Bestandteile wurden vermehrt in den Gräbern der frühbronzezeitlichen Bevölkerung deponiert. Zudem spricht ein weiträumig kohärentes Bestattungsritual im Nordpontikum und den daran angrenzenden Regionen für weitreichende kulturelle Kontakte. Paläoklimatische Untersuchungen machen außerdem für den Zeitraum 
ab dem Ende des 4. einen Wandel in den klimatischen Verhältnissen wahrscheinlich, der mit einem Aridisierungsprozess im folgenden 3. Jt.v. Chr. einherging. All dies sind Gründe, die zu Änderungen in der Lebensweise geführt haben könnten, möglicherweise in Verbindung mit erhöhter Mobilität. Als Vergleichsbasis für das 4. und 3. Jahrtausend dienten Proben, die in das I. Jt.v. Chr. datieren, denn für diese Zeit gibt es nicht nur archäologische sondern auch schriftliche Quellen, die von einer erhöhten Mobilität der skythischen Gemeinschaften und kulturell verwandter Stämme zeugen.

Während Kapitel I einer kurzen Projektskizze sowie der chronologischen und kulturellen Einordnung der Studie dient, folgt in Kapitel 2 eine Charakterisierung der für diese Studie relevanten archäologischen Kulturen sowie eine Übersicht über die angewandten Methoden und die Wahl des Probenmaterials. Kapitel 3 ist der Vorstellung der Fundorte gewidmet, die die Proben für die stabilen Isotopenanalysen lieferten. Die Selektion der Fundorte, die nicht in den Händen der Verfasserin lag, sollte einen repräsentativen Querschnitt durch Raum und Zeit bieten. Bedauerlicherweise wurde nicht auf das Vorhandensein statistisch relevanter Probenmengen geachtet. Zudem lagen von keinem Fundort einheitliche Verteilungen von Alters- und Geschlechtsgruppen sowie chronologischen Perioden vor, Umstände, die eine Evaluation der Analyseergebnisse erheblich beeinträchtigten.

Die eigentliche Untersuchung war in die beiden großen Teilbereiche Mobilität und Migration sowie Ernährung gegliedert, welche aufgrund unterschiedlicher Materialbasen als zwei nur bedingt miteinander verbundene Studien angesehen werden können. Die Untersuchung zu Mobilität und Ernährung

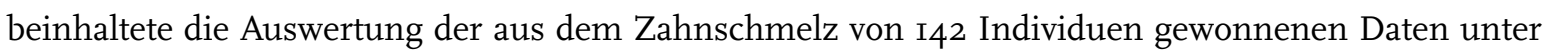
Anwendung der Strontium- $\left({ }^{87} \mathrm{Sr} /{ }^{86} \mathrm{Sr}\right)$ und Sauerstoffisotopenanalyse $\left(\delta^{18} \mathrm{O}\right)$. Die Anwendung von Kohlenstoff- $\left(\delta^{{ }^{13}} C\right)$ und Stickstoffisotopenanalysen $\left(\delta^{15} \mathrm{~N}\right)$ am Knochenmaterial 54 menschlicher Individuen von ausgewählten Fundorten aus dem West- und dem Nordpontischen Raum sowie dem Kubangebiet ermöglichte zudem einen interregionalen diachronen Vergleich zum Ernährungsverhalten.

In der archäologischen Forschung werden Mobilitätstheorien zwar häufig erwähnt, nur sporadisch finden sie jedoch tatsächlich Eingang in die Evaluation des Fundmaterials. Auch in der vorliegenden Studie erwies es sich als schwierig, die zu Beginn des Kapitels 4 vorgestellten Theorien anzuwenden, da eine Reihe von Unsicherheitsfaktoren vorliegen: Die kulturellen Grenzen der äneolithischen Steppenkulturen und der Regionalgruppen von Jamnaja- und Katakombengrabkulturen sind verhältnismäßig vage und werden häufig diskutiert. Die wirtschaftlichen Grundlagen dieser Kulturen implizieren ebenfalls Unsicherheiten. So ist zwar sicher, dass eine auf Rinder basierende Viehhaltung die dominierende ökonomische Grundlage bildete, mit der ein gewisser Grad an Mobilität verbunden war, ungewiss bleibt jedoch, in welchem Maße eine solche Mobilität vorlag. Ebenso sind die Mobilitätsradien und -ausrichtungen unbekannt, was unter anderem durch die wenigen gegrabenen Siedlungen begründet ist. Einige Siedlungen implizieren eine temporäre, saisonale Nutzung wie z. B. in der Region nordwestlich des Kaspischen Meeres, andererseits liegen auch Hinweise für permanente oder längerfristig genutzte Siedlungen vor, so z. B. am Unteren Dnjepr. Es fehlen jedoch prägnante Indikatoren für mögliche Mobilitätszyklen, wie z.B. zirkuläre oder an Ressourcen orientierte Wanderungswege. Des Weiteren stellt sich die Frage nach der Relevanz einer Referenzprobennahme in der Umgebung eines Grabhügels, für den ungeklärt ist, inwieweit dieser Platz deckungsgleich mit dem letzten Aufenthaltsort von auf irgendeine Weise mobil lebenden Gemeinschaften ist.

Die Kombination aus Strontium- und Sauerstoffisotopenanalysen, welche von verschiedenen Faktoren abhängen, ermöglichte trotz einiger Überlappungen eine isotopenchemische Differenzierung der meisten Mikroregionen und vereinfachte so die Identifizierung von Ausreißern. Die Ergebnisse der Strontiumisotopenanalyse variierten und zeigten auf der einen Seite sehr einheitliche Isotopen- 
verhältnisse der menschlichen Individuen eines Fundortes, wie z. B. in Benkovski (Bulgarien), Nevskoe (Ukraine) und Olennij (Russland), während aus anderen Fundorten eine große Variabilität in den ${ }^{87} \mathrm{Sr} /{ }^{86} \mathrm{Sr}$-Werten vorlag, z. B. in Ovčarci (Bulgarien), Kirovograd (Ukraine) und an den Fundorte aus dem Mittleren Wolgagebiet (Russland). Die Ergebnisse der Sauerstoffisotopenanalyse stimmten mit diesen nur bedingt überein. Fundorte, die hinsichtlich ihrer ${ }^{87} \mathrm{Sr} /{ }^{86} \mathrm{Sr}$-Werte wenig bemerkenswert waren, lieferten teilweise eine große Variabilität in ihren $\delta^{18} \mathrm{O}-W e r t e n$, so z.B. in Vinogradnoe und Nevskoe (Ukraine), während Fundorte mit heterogenen ${ }^{87} \mathrm{Sr} /{ }^{86} \mathrm{Sr}$-Ergebnissen hinsichtlich $\delta^{18} \mathrm{O}$ nur geringfügig variierten, z.B. in Kirovograd (Ukraine). Erst die Kombination der beiden Methoden ermöglichte in vielen Fällen die Identifizierung von Mobilitätsmustern. Jedoch kann nicht definitiv geklärt werden, ob die Unterschiede mit der variierenden Datenbasis oder durch tatsächlich größere Mobilitätsradien begründet werden können, da verhältnismäßig homogene Isotopenverhältnisse mehrfach mit Kurganen korrelierten, die von nur einer Kultur belegt wurden, so z.B. in Nevskoe (Ukraine), Olennij und Suchaja Termista II (Russland), während weit streuende Isotopenresultate oftmals mit einem diachron belegten Fundplatz einhergingen, z. B. in Kirovograd und Pesčanka (Ukraine). Es liegen jedoch auch Gegenbeispiele vor. Unterschiedliche Strategien bei der Referenzprobenauswahl erschwerten einen intra- und interregionalen Vergleich. Unter Vernachlässigung der variierenden Vertrauensbasen konnte jedoch vorgeschlagen werden, dass die Ergebnisse der äneolithischen und jamnajazeitlichen Proben in den westlichen Untersuchungsregionen weiter streuen als die in anderen Gebieten. Nur wenig Variation konnte für die Frühe Katakombengrabkultur konstatiert werden. Die Ergebnisse suggerieren einen potentiellen Wechsel in den Isotopenmustern, der möglicherweise mit dem einsetzenden Aridisierungsprozess und bzw. oder einem ökonomischen Wandel am Ende des 4. Jahrtausends v. Chr. in Verbindung steht. Für herausstechende Individuen, wie z. B. solche, die mit einem Wagen bestattet wurden, konnte isotopenchemisch kein erhöhter Mobilitätsgrad nachgewiesen werden.

Die relativ homogenen ${ }^{87} \mathrm{Sr} /{ }^{86} \mathrm{Sr}$ - und $\delta^{18} \mathrm{O}$-Daten der skythenzeitlichen Proben aus dem Nordpontikum können entweder als Nachweis eingeschränkter Ortswechsel interpretiert werden oder als Beweis dafür angesehen werden, dass die geringen geologischen und klimatischen Unterschiede in eine begrenzte isotopenchemische Variabilität resultieren. Einheitliche Mobilitäts- und Ernährungsmuster auf Basis einer zusammengehörigen Siedlungsgemeinschaft erscheinen aufgrund des Vorhandenseins mehrerer Fundorte unwahrscheinlich. Eine erhöhte Mobilität, die möglicherweise mit Transhumanz in Verbindung gebracht werden kann, deutete sich durch die größeren ${ }^{87} \mathrm{Sr} /{ }^{86} \mathrm{Sr}$ - und $\delta^{18} \mathrm{O}$-Spannen und Standardabweichungen der skythenzeitlichen Menschen und Tiere aus dem kasachischen Altaigebirge an. Auch die Isotopenverteilungen in den Tierzähnen einer in Kapitel 5 diskutierten dreifachen Rinderdeponierung von Zauschwitz (Deutschland), die der Kugelamphorenkultur zugeordnet wird und damit zeitliche und wirtschaftliche Parallelen zur Jamnajakultur aufweist, sprechen zumindest teilweise für das Praktizieren von Transhumanz. In diesem Zusammenhang erbrachten zusätzliche ${ }^{87} \mathrm{Sr} /{ }^{86} \mathrm{Sr}$-Laserablationen an diesen Zähnen einen beeindruckenden Mehrwert hinsichtlich des Nachweises von regulärer Mobilität.

Migrationen ganzer Gemeinschaften der äneolithischen und frühbronzezeitlichen Steppenkulturen in die Regionen westlich und südwestlich des Verbreitungsgebietes lassen sich auf der vorhandenen Datenbasis mit Hilfe von Strontium- und Sauerstoffisotopenanalysen weder zweifelsfrei bestätigen noch eindeutig dementieren. Einige Individuen in jamnajazeitlichen Hügelgräbern in Ungarn und möglicherweise ebenso die weit verteilten und partiell mit den Erwartungswerten für das Nordpontikum übereinstimmenden Isotopenverhältnisse einiger Individuen in den frühbronzezeitlichen Kurga- 
nen Bulgariens, sprechen in Kombination mit dem archäologischen Befund jedoch sehr wahrscheinlich für die Einwanderung von Individuen. Dagegen wurden suggerierte Migrationen anderer Individuen, für die ebenfalls ein fremdes Bestattungsritual nachgewiesen ist, welches Charakteristika mit den im Nordpontikum ansässigen Kulturen aufweist, isotopenchemisch nicht bestätigt. Aufgrund der relativ geringen Datenbasis und der Tatsache, dass die einheimischen Bestattungsrituale nur bedingt archäologisch charakterisiert sind, müssen jedoch weitere Analysen abgewartet werden, die die vorläufigen Interpretationen bestätigen oder revidieren werden.

Zusätzlich zu den Untersuchungen zu Mobilität und Migration wurden für eine Studie zur Rekonstruktion der Ernährung Kohlenstoff- und Stickstoffisotopenanalysen an menschlichem Skelettmaterial, genauer an 53 menschlichen Knochen und acht Zähnen, angewandt. Das Probenmaterial stammte aus drei Fundorten in Bulgarien, vier Fundplätzen in der Ukraine und einem Fundort im russischen Kubangebiet. Statistische Tests bezeugten eine positive Korrelation zwischen $\delta^{13} \mathrm{C}$ und $\delta^{15} \mathrm{~N}$, die zudem mit der geographischen Lage und den Klimaverhältnissen an den Fundorten korreliert. Eine Ernährung auf $\mathrm{C}_{3}$-Pflanzenbasis bildete die Grundlage aller untersuchten Individuen, wobei sich die Daten der bulgarischen menschlichen Individuen deutlich von denen in der Ukraine und im Kubangebiet unterschieden. Eine Verschiebung um $3 \%$ in $\delta^{\text {Is }} \mathrm{N}$ zeigte sich zwischen Herbivoren von bulgarischen und ukrainischen Fundorten und solchen in Südrussland sowohl in Vergleichsstudien als auch in den wenigen Faunenreferenzen, die im Rahmen dieser Studie untersucht werden konnten. Diese Verschiebung kann mit unterschiedlichen klimatischen Verhältnissen erklärt werden. In Übereinstimmung mit früheren Studien, in denen leichte stabile Isotope auf prähistorische menschliche Individuen im Westeurasischen Steppengürtel angewandt wurden, kann vorgeschlagen werden, dass Fisch und andere Süßwassertiere ein zusätzlicher Bestandteil der Ernährung der äneolithischen und bronzezeitlichen Bevölkerung in diesen Regionen war, während die $\delta^{13} \mathrm{C}$ - und $\delta^{15} \mathrm{~N}$-Isotopenverhältnisse der bulgarischen Individuen in dieser Studie keinen Hinweis auf einen erhöhten Verzehr von Süßwasserressourcen gaben. Dagegen lassen die Isotopensignaturen der frühbronzezeitlichen Individuen in dieser Region auf einen nicht unwesentlichen Einfluss von $\mathrm{C}_{4}$-Pflanzen schließen. Dies könnte als Indiz für den Anbau von $\mathrm{C}_{4}$-Pflanzen wie z. B. Hirse gedeutet werden. Diese Pflanzen sind im Nordpontikum für das Äneolithikum und die frühe Bronzezeit nicht sicher belegt und ihr Anbau spielt erst ab der Späten Bronzezeit und in der folgenden Eisenzeit eine größere Rolle. Statistische Tests konnten aufgrund der vorliegenden Datenbasis lediglich mit großen Einschränkungen durchgeführt werden. Obwohl keine einheitliche chronologische Tendenz vorlag, konnten Unterschiede zwischen den Individuen der Jamanja- und der Frühen Katakombengrabkultur und in Vinogradnoe zudem zwischen Früher und Entwickelter Katakombengrabkultur erkannt werden. Marginale Differenzen ließen die $\delta^{\mathrm{r} 3 \mathrm{C}} \mathrm{C}$ und $\delta^{15} \mathrm{~N}$-Isotopenverhältnisse von Kindern und Erwachsenen erkennen, welche auf eine fleisch- oder fischreichere Ernährung schließen lassen, geschlechtsspezifische Unterschiede lagen dagegen nicht vor. Zusammenfassend lässt sich sagen, dass die großräumig angelegte Untersuchung das Erkennen von Ernährungsunterschieden in und zwischen den einzelnen Regionen des Untersuchungsgebietes ermöglichte. Die dominierende Nahrungsgrundlage der äneolithischen, früh- und mittelbronzezeitlichen Steppenbevölkerung bildeten $\mathrm{C}_{3}$-Pflanzen, Herbivoren, die sich von diesen ernährten, und die Produkte dieser Tiere, gleichwohl werden aber auch Süßwasserprodukte eine Rolle gespielt haben. Die Unterschiede in den $\delta^{13} \mathrm{C}$ - und $\delta^{15} \mathrm{~N}$ - Isotopenverhältnissen der nord- und westpontischen Individuen lassen sich zum Teil mit variierenden klimatischen Bedingungen und unterschiedlichem Bewaldungsgrad erklären. Zudem ist ein unterschiedlich großer Einfluss von Süßwasserressourcen wahrscheinlich. Für eine fundierte Aussage zu den Ernährungsgewohnheiten der diskutierten archäologischen 
Gruppen bedarf es jedoch einer Erweiterung der Probenmenge an menschlichen Individuen als auch an der lokalen Fauna und infolgedessen der Rekonstruktion eines ausgedehnten Nahrungsnetzes, welches die Grundlage für Mixing models bilden kann.

Bereits zu Beginn des Projektes zeichnete sich ab, dass die Anwendung von Multi-Isotopenanalysen auf ein räumlich und zeitlich extrem weit gefasstes Gebiet diffizil werden würde, und es ist zwingend notwendig eine Beprobungsstrategie zu verfolgen, die trotz dieses Rahmens denselben Qualitätsanforderungen wie in Untersuchungen von einzelnen Fundorten folgt. Statistisch relevante Probenmengen menschlicher Individuen, aber auch eine profunde Untersuchung möglicher Nahrungs- und Trinkwasserquellen sind erforderlich um fundierte Aussagen treffen zu können. Daher empfiehlt sich anstelle einer regellosen Probenselektion die Auswahl weniger Grabhügel an geologisch und klimatisch verheißungsvollen Plätzen, die auf statistisch relevanter Datenbasis eine diachrone Beprobung menschlicher Individuen ermöglichen. Nicht weniger wichtig ist eine detaillierte isotopenchemische Charakterisierung der näheren Umgebung. Auf der vorliegenden Datenbasis konnte gezeigt werden, dass das Arbeitsgebiet isotopenchemisch differenzierbar ist, und obwohl mit dem vorhandenen Probenmaterial nur vorläufige Aussagen gemacht und wahrscheinliche Tendenzen angesprochen werden konnten, bietet die Untersuchung einen extrem breit gefächerten Grundstock an Analysedaten, auf den zukünftige Studien aufbauen können.

Данное исследование объединило методы археологии и естественных наук с целью освещения поведения населения западноевразийских степей в отношении мобильности, а также характера его питания в период с 3500 по 300 гг. до н. э. Речь шла об одном из подпроектов исследовательской группы A II Spatial effects of technological innovations and changing ways of life в рамках междисциплинарного научно-исследовательского проекта Тороі. С этой целью были выбраны репрезентативные микрорегионы, а также отдельные местонахождения в Западном Причерноморье (в Болгарии), Карпато-Балканском регионе (в Венгрии), Северном Причерноморье (в южной части центральной Украины и в восточной Украине), в России (на Кубани, в Северо-Восточном Прикаспие и в Среднем Поволжье), а также в казахстанской части Алтая. Из названных регионов были отобраны человеческие индивиды, которые датируются второй половиной IV, III и I тыс. до н. э. и принадлежат к энеолитическим степным культурам, ямной и катакомбной культурам бронзового века, а также культурным группам, относящимся к скифскому периоду. На их зубах и костях были проведены мульти-изотопные анализы, а именно анализы изотопов стронция ${ }^{87} \mathrm{Sr} /{ }^{86} \mathrm{Sr}$, кислорода $\delta^{18} \mathrm{O}$, азота $\delta^{15 \mathrm{~N}}$ и углерода $\delta^{13} \mathrm{C}$. Вторым центральным вопросом, наряду с разбором и оценкой результатов, полученных для отдельных местонахождений, и предложением проекта реконструкции возможных поведенческих моделей этих сообществ в отношении мобильности и питания, был вопрос о том, возможно ли ввиду инновационных процессов констатировать повышенную мобильность. Для решения этой задачи были исследованы периоды до и после 3000 г. до н. э. с целью выявления возможных различий в распределениях соотношений изотопов. В связи с ямной культурой приблизительно на рубеже IV-III тыс. до н. э. произошёл переход к животноводству, специализированному на содержании крупного рогатого скота, и в погребения населения раннего бронзового века стали чаще помещать такие средства передвижения, как повозки или их составные части. К тому же сходный погребальный обряд на значительной территории Северного Причерноморья и прилегающих к нему регионов свидетельствует об обширных культурных контактах. Кроме этого, исходя из палеоклиматических исследований, 
вероятны изменения климатических условий в период начиная с IV. тыс. до н. э., которые сопровождались процессом аридизации в последующем III тыс. до н. э. Всё перечисленное является причинами, которые могли повлечь за собой изменение образа жизни, связанного, возможно, с повышенной мобильностью. Основой для сравнения послужили образцы, датируемые I тыс. д Сн. э., так как для этого времени имеются не только археологические, но и письменные источники, свидетельствующие о повышенной мобильности скифских сообществ и культурно им родственных племён.

В то время как глава I служит краткому описанию проекта, а также определению хронологических и культурных рамок исследования, в главе 2 представлены существенные для этого исследования археологические культуры, а также даны обзор применённых методов и пояснение принципов отбора материала для анализов. Глава 3 посвящена ознакомлению с местонахождениями, из которых были взяты образцы для анализов стабильных изотопов. Отбор местонахождений, на который автор исследования не имела возможности оказать влияние, должен был предоставить репрезентативный обзор во времени и пространстве, хотя, к сожалению, при этом не была принята во внимание необходимость наличия статистически существенных количеств образцов. Кроме этого, ни для одного из местонахождений не имеется равномерного распределения возрастно-половых групп, а также хронологических периодов, что усложняет обобщение полученных результатов.

Само исследование было поделено на две больших подчасти, а именно “мобильность и миграция” и “палеодиета”, которые лишь условно могут рассматриваться как два связанных друг с другом исследования, так как их основополагающие материальные базы различны. Исследование мобильности и питания включало в себя анализ данных, полученных из зубной эмали I42 индивидов, применяя анализ изотопов стронция $\left({ }^{87} \mathrm{Sr} /{ }^{86} \mathrm{Sr}\right)$ и кислорода $\left(\delta^{18} \mathrm{O}\right)$. Применение анализов изотопов углерода $\left(\delta^{13} \mathrm{C}\right)$ и азота $\left(\delta^{15} \mathrm{~N}\right)$ к остеологическому материалу от 54 человеческих индивидов из избранных местонахождений западно- и северопричерноморского региона, а также Кубани дало возможность провести межтерриториальные диахронные сравнения характера питания.

Несмотря на то что теории мобильности в археологических исследованиях и упоминаются часто, они всё же редко принимаются во внимание при обобщающем анализе археологического материала. И в данном исследовании оказалось сложным применить представленные в начале главы 4 теории, так как присутствовал ряд факторов, влияющих на достоверность. Культурные границы степных энеолитических культур и региональных групп ямной и катакомбной культур сравнительно неопределённые и часто бывают предметом дискуссий. Также таит неясности экономическая основа ямной и катакомбной культур. Хотя и достоверно известно, что преобладающим экономическим основанием этих культур было животноводство, специализирующееся на крупном рогатом скоте, с чем была связана определённая степень мобильности, всё же остаётся неясным, в какой мере эта мобильность проявлялась. Также неизвестны радиусы и направления передвижений, причиной чего, в частности, является малое количество раскопанных поселений. Для некоторых поселений можно предположить временное, сезонное использование, с другой стороны, есть признаки, указывающие на бывшие постоянно или на протяжении более длительного периода в использовании поселения, как, например, в Нижнем Поднепровье. Однако отсутствуют выразительные индикаторы возможных циклов передвижений, как, например, циркулярные или ориентирующиеся на ресурсы пути миграций. Следующим вопросом является вопрос о том, насколько существен контрольный замер в окрестностях кургана, так как не выяс- 
нено, является ли это место тождественным с местом последнего пребывания сообществ, живущих в какой-либо мере мобильно.

Сочетание анализов изотопов стронция и кислорода, которые не зависимы друг от друга, а зависят от различных факторов, дало возможность, несмотря на некоторые наложения, провести изотопно-химическое дифференцирование большинства микрорегионов и облегчило таким образом распознавание экстремальных значений. Результаты анализа изотопов стронция варьировали и проявили, с одной стороны, очень единообразные соотношения изотопов для человеческих индивидов одного местонахождения, как, например, Бенковски (Болгария), Невского (Украина) и Оленнего (Россия), в то время, как для других местонахождений - например, Овчарци (Болгария), Кировограда (Украина) и местонахождений Среднего Поволжья (Россия) - было налицо широкое варьирование значений ${ }^{87 \mathrm{Sr}} /{ }^{86} \mathrm{Sr}$. Результаты анализа изотопов кислорода соответствовали им лишь в ограниченной мере. Местонахождения, которые были мало примечательными относительно значений ${ }^{87} \mathrm{Sr} /{ }^{86} \mathrm{Sr}$, показали отчасти широкую вариативность в их $\delta^{18} \mathrm{O}$-значениях, как, например, Виноградное и Невское (Украина), в то время, как местонахождения с гетерогенными результатами по ${ }^{87} \mathrm{Sr} /{ }^{86} \mathrm{Sr}$ варьировали относительно б$^{18} \mathrm{O}$ незначительно, например, Кировоград (Украина). Лишь сочетание обоих методов дало во многих случаях возможность распознать модели мобильности. Однако определённо не может быть выясненым, могут ли варьирующая база данных или действительно бо́льшие радиусы передвижений являться причиной этих различий, так как сравнительно гомогенные соотношения изотопов во многих случаях коррелируют с теми курганами, которые содержат погребения лишь одной культуры, как, например, в Невском (Украина), Оленнем и в могильнике Сухая Термиста II (Россия), в то время, как широко разбросанные результаты анализов изотопов часто были связаны с местонахождением, содержащим погребения разновременных культур, например, в Кировограде и Песчанке (Украина). Но есть и примеры, свидетельствующие об обратном. Различные стратегии выбора материала для контрольных замеров усложняли внутри- и межрегиональное сопоставление. Не принимая во внимание то обстоятельство, что образцы для контрольных замеров были различными в отношении степени полагаемого на них доверия, можно было предположить, что результаты анализов образцов периодов энеолита и раннего бронзового века в западной части территории исследования разбросаны шире, чем в других областях. Лишь незначительные вариации могли быть констатированы для ранней катакомбной культуры. Эти результаты наводят на мысль о возможном изменении моделей изотопов на переходе к раннему этапу катакомбной культуры, которое, как можно предположить, связано с начинающимся процессом аридизации и/ или экономическими преобразованиями в конце IV тыс. до н. э. Для выделяющихся личностей, например, погребённых с повозкой, изотопно-химически не была доказана повышенная степень мобильности. Сравнительно однородные данные по ${ }^{87} \mathrm{Sr} /{ }^{86} \mathrm{Sr}$ и $\delta^{\text {I8 }} \mathrm{O}$ для образцов скифского периода из Северного Причерноморья могут быть интерпретированы или как свидетельство относительно ограниченной перемены местожительства, или же как доказательство того, что из незначительных геологических и климатических различий следует ограниченная изотопно-химическая вариабельность. Объяснением таких единообразных моделей мобильности и питания не может быть сплочённость сообщества поселений, так как анализы были проведены для образцов из различных местонахождений. Повышенная мобильность, которая, возможно, связана с транзюмансом, вырисовалась из широких промежутков ${ }^{87} \mathrm{Sr} /{ }^{86} \mathrm{Sr}$ и $\delta^{18} \mathrm{O}$, а также высоких стандартных отклонений для людей и животных скифского времени из казахстанского Алтая. Распределения изотопов в зубах животных рассматриваемого в главе 5 тройного трупоположения 
быков из Цаушвитца (Германия), которое относят к культуре шаровидных амфор, проявляющей хронологические и экономические параллели к ямной культуре, свидетельствуют по меньшей мере частично о практицировании транзюманса. Дополнительные анализы ${ }^{87 \mathrm{Sr}} /{ }^{86} \mathrm{Sr}$ посредством лазерной абляции на тех же зубах предоставили впечатляющие добавочные результаты относительно доказательства обыденной мобильности.

Миграции степных культур периодов энеолита и раннего бронзового века в регионы к западу и юго-западу от области их распространения не могут быть на основании имеющихся данных с помощью анализов изотопов стронция и кислорода ни безусловно подтверждены, ни однозначно отвергнуты. Некоторые погребённые в курганах ямного времени в Венгрии, а, возможно, также и соотношения изотопов некоторых индивидов из курганов раннего бронзового века в Болгарии, показатели которых широко разбросаны и частично соответствуют ожидаемым для Северного Причерноморья соотношениям, свидетельствуют в сочетании с археологическим контекстом с большой вероятностью о переселении индивидов в эти регионы. С другой стороны, предположение о том, что миграции могли иметь место, не подтвердились с помощью изотопно-химических анализов для других индивидов с чужеродным ритуалом погребения, которому присущи черты, характерные для культур Северного Причерноморья. В силу относительно небольшой базы данных и того обстоятельства, что локальным погребальным обрядам дана лишь недостаточная археологическая характеристика, необходимо всё же дождаться дальнейших анализов, которые позволят подтвердить или исправить предварительные интерпретации. Помимо изучения мобильности и миграций, анализы изотопов углерода и азота были применены на человеческом скелетном материале, а точнее 53 человеческих костях и восьми зубах, для реконструкции питания. Материал для анализов происходил из трёх местонахождений в Болгарии, четырёх местонахождений в Украине и одного на Кубани в России. Статистические тесты удостоверили позитивную корреляцию между показателями $\delta^{13} \mathrm{C}$ и $\delta^{15} \mathrm{~N}$, и эти показатели в свою очередь коррелируют с географическим положением и климатическими условиями по месту находки. Питание на основании С3-растений составляло основу диеты всех исследованных индивидов, причём данные, полученные для человеческих индивидов Болгарии, чётко отличались от данных для образцов из Украины и Кубани. Смещение показателей $\delta^{15} \mathrm{~N}$ на 3 \%о обнаружено при сопоставлении травоядных из болгарских и украинских местонахождений с травоядными юга России как в сравнительных исследованиях, так и в немногих ссылках на фауну, которые могли быть рассмотрены в рамках этого исследования. Это смещение можно объяснить различными климатическими условиями. В соответствии с предыдущими исследованиями, в которых анализ лёгких стабильных изотопов был применён к доисторическим человеческим индивидам западноевразийского степного пояса, можно предположить, что рыба и другие пресноводные животные были дополнительной составляющей частью диеты энеолитического населения и населения бронзового века этих регионов, в то время, как соотношения изотопов $\delta^{13} \mathrm{C}$ и $\delta^{15} \mathrm{~N}$ для индивидов из местонахождений Болгарии не засвидетельствовали в данном исследовании об употреблении в пищу пресноводных рессурсов. Напротив, изотопные шифры населения раннего бронзового века этого региона позволяют сделать вывод о немаловажном влиянии С4-растений. Это могло бы быть свидетельством выращивания С4-растений, например, проса, которые для Северного Причерноморья периодов энеолита и раннего бронзового века достоверно не засвидетельствованы, и их выращивание имеет бо́льшее значение лишь начиная с позднего бронзового, а также в последующем железном веке. 
Статистические тесты могли быть проведены на основании имеющейся в наличии базы даных лишь с большими ограничениями. Хотя единообразной хронологической тенденции и не наблюдалось, могли быть распознаны различия между индивидами ямной и раннекатакомбной культуры, а в Виноградном к тому же и между представителями ранней и развитой фазы катакомбной культуры. Соотношения изотопов $\delta^{13} \mathrm{C}$ и $\delta^{15} \mathrm{~N}$ детей и взрослых позволили обнаружить лишь незначительные различия, что позволяет сделать вывод о преобладании мяса или рыбы в рационе, в то время как гендерных различий не наблюдалось. Широкомасштабно спланированное исследование позволило распознать в границах его территории внутри- и межрегиональные различия в рационе питания, причиной которых могут быть климатические изменения. Преобладающую основу рациона различных групп степного населения энеолита, раннего и среднего бронзового века составляли С3-растения, травоядные животные, питающиеся этими растениями, а также продукты от этих животных. В то же время значимыми могли быть и пресноводные продукты. Различия в соотношениях изотопов $\delta^{13} \mathrm{C}$ и $\delta^{15} \mathrm{~N}$ северо- и западнопричерноморских индивидов можно частично объяснить изменяющимися климатическими условиями, так как не только люди, но и травоядные животные обнаруживают различные соотношения изотопов. Кроме того, вероятно, что влияние пресноводных рессурсов было различным по значимости. Для обоснованного утверждения о привычках питания обсуждаемых археологических групп необходимыми являются расширение количества образцов для анализов как от людей, так и от локальных животных, и вследствие этого реконструкция расширенной сети питания, которые могут сформировать основу для моделей смешения изотопов (Mixing models).

Уже к началу работы над проектом обозначилось, что применение мульти-изотопных анализов для крайне обширной территории с очень широким хронологическим диапазоном может быть связано со сложностями. Настоятельно необходимо придерживаться такой стратегии отбора и анализа образцов, которая, несмотря на широкие рамки исследования, отвечает тем же требованиям к качеству, что и при проведении анализов для отдельных местонахождений. Для обоснованных утверждений необходимы статистически существенные количества образцов от человеческих индивидов, а также основательное исследование возможных источников питания и питьевой воды. Поэтому вместо несистематического отбора проб рекомендуется выбрать небольшое количество курганов, расположенных в геологически и климатически многообещающих местах, которые позволили бы осуществить диахронический анализ человеческих индивидов на статистически существенном основании. Не менее важной является подробная изотопно-химическая характеристика ближайших окрестностей. На основе имеющихся данных представилось возможным показать, что территория исследования дифференцируема с изотопно-химической точки зрения. Несмотря на то, что на основе имеющихся в наличии образцов для испытаний могут быть сделаны лишь предварительные высказывания и рассмотрены вероятные тенденции, исследование предоставляет крайне обширный фундамент данных анализов, на основе которых могут быть построены дальнейшие исследования.

Translation: N. Chub 